Results Subfertility was reported by $9.1 \%$ of the women $(n=719)$. Prevalence was $8.7 \%$ among primigravidae and $9.5 \%$ in multigravidae ( $84 \%$ of the multigravidae were multipara). Almost $2 / 3$ sought for medical help. Among young primigravidae, subfertility decreased with education ( $>12$ vs $\leq 6$ years: OR $0.2095 \%$ CI 0.11 to 0.39), and was lower among single (OR $0.2795 \%$ CI 0.12 to 0.59 ) and smokers (OR $0.60 ; 95 \%$ CI 0.40 to 0.94 ). Only education presented a significant association with subfertility in older primigravidae. Within multigravidae higher education increased the odds. Maternal childhood conditions were no longer significant after adjustment for current social circumstances.

Conclusions Age and the number of previous births modified the effect of social conditions on subfertility which was stronger among younger and primigravidae women.

\section{SP3-90 HOSPITALISATION TRENDS IN PUBLIC PSYCHIATRY HOSPITAL (2005-2010)}

doi:10.1136/jech.2011.1429760.90

H Oliveira, ${ }^{*} J$ Aquino, A Ferreira, C Verçosa, M Rezende, H Barros. Instituto Raul Soares/FHEMIG, Belo Horizonte, Minas Gerais, Brazil

Introduction The study describes and analyzes the hospitalisations due to mental disorders during 2005-2010 in public psychiatric hospital in Belo Horizonte, Brazil.

Methods We conducted a retrospective study of hospitalisation data using the EPI-INFO. Length of hospital stay, main psychiatric diagnoses (ICD-10, WHO) and sociodemographic characteristics were analysed.

Results The predominant hospitalisations group were from men with 20 to 50 years old and patients with mental disorders related to substance use. There were a decreased of total hospitalisations, length of hospital stay and percentual of patients with ICD-10 diagnoses F20-F29. There were increased in the percentual of patients with ICD-10 diagnoses F10-19. Remained constant the percentual of admissions to patients from different places of country. Conclusion There was a significant decrease in the hospital stay, indicating better quality of hospital care, availability of resources therapeutic, increased care network to outpatients and access to psychopharmacological treatment. But there are a important increased admissions of patients with mental disorders related to substance use. The Psychiatric hospitalisation is important to psychiatric care network and there are changes the profile of diagnoses that need to be evaluated in the planning of public services mental health.

\section{SP3-91 ASSOCIATED FACTORS WITH CHANGES IN SOCIAL SUPPORT NETWORK FOR THE OLDER ADULTS IN 6 YEARS}

doi:10.1136/jech.2011.1429760.91

M L Lebrão,* S Alencar, Y Duarte. University of Sao Paulo, Sao Paulo, Sao Paulo, Brazil

Introduction Ageing process brings people living with the physical and functional limitations and multiple chronic diseases, added to significant emotional aspects that increase order's demand of their social network.

Objective Analyze changes during the period of 6 years (2000-2006) in the social support network of older adults of Sao Paulo-Brazil.

Methods Data comes from a longitudinal survey-SABE (Health, Well-being and Ageing), which began in 2000 with a multistage clustered sample of 2143 people aged $\geq 60$ years-old in Sao PauloBrazil. In the second wave 1115 elderly were re-interviewed. Associated factors were analysed in multinomial logistic regression.

Results Older adults refer more instrumental support at home and among their children. The support from outside the home of other relatives and friends showed present but fluctuates over time of the study. Physical and functional conditions, self-reported health were indicators of maintaining the exchange of support with all network over time but the worsening in these conditions was accompanied by more support available for elderly.

Conclusions The informal social support network is required only for instrumental support needs that are not served by the formal support network. The emotional aspects influencing independence and autonomy when they are not adequately considered.

\section{SP3-92 INFLUENZA LIKE ILLNESS MONITORING BY TELEPHONE SURVEY IN BRAZIL, VIGITEL, 2010}

doi:10.1136/jech.2011.142976o.92

${ }^{1} \mathrm{~L}$ Souza, ${ }^{*} \mathrm{P}$ Reis, ${ }^{2} \mathrm{~B}$ Iser, ${ }^{2,3} \mathrm{R}$ Yokota, ${ }^{1} \mathrm{~W}$ Almeida, ${ }^{4} \mathrm{R}$ Bernal, ${ }^{2} \mathrm{D}$ Malta, ${ }^{1} \mathrm{~W}$ Oliveira. ${ }^{1}$ General Coordination of Transmissible Diseases, Department of Epidemiological Surveillance, Ministry of Health, Brasilia, Distrito Federal, Brazil; ${ }^{2}$ General Coordination of NonCommunicable Diseases and Injuries, Ministry of Health, Brasilia, Distrito Federal, Brazil; ${ }^{3}$ Brazilian Field Epidemiology Training Program, Ministry of Health, Brasilia, Distrito Federal, Brazil, " ${ }^{4}$ Public Health Faculty, University of São Paulo, São Paulo, São Paulo, Brazil

Introduction In Brazil, after influenza pandemic in 2009, influenza surveillance system was restricted to cases who sought healthcare assistance within severity signs and symptoms.

Objective To estimate the prevalence of influenza like illness (ILI) cases, ILI cases who sought healthcare assistance, with pandemic influenza H1N1 2009 (pH1N1) medical suspicion and oseltamivir prescription among the $\mathrm{pH} 1 \mathrm{~N} 1$ suspected cases by telephone survey. Method In 2010, an influenza module of four questions was firstly included in the Telephone-based System for the Surveillance of Risk and Protective Factors for Chronic Diseases (Vigitel). In this study, data from January to November 2010 were analysed. The proportion of cases stratified by sociodemographic characteristics and Brazilian geographic region was weighted with data from the National Survey with Household Sampling, 2008.

Result The prevalence of ILI cases was $31.2 \%$ (95\% CI $30.2 \%$ to $32.1 \%$ ) in the period studied and it was higher among women, young adults (18-29 years of age) and individuals with higher education level. Northern Brazil presented the highest prevalence (36.8\% of ILI cases; $95 \%$ CI 35.3 to $38.3 \%$ ) of ILI cases. Need for healthcare assistance was reported by $26.8 \%$ (95\% CI 25.1 to $28.5 \%$ ) of ILI cases. Among ILI cases $2.5 \%$ (95\% CI 1.8 to $3.4 \%$ ) reported pandemic influenza H1N1 2009 (pH1N1) suspicion. Oseltamivir prescription was reported by $4.9 \%$ (95\% CI 1.1 to $8.7 \%$ ) pH1N1 suspected cases. Conclusion The results of Vigitel supported the influenza surveillance in Brazil as it provided timeliness and useful information, which was not collected by the traditional surveillance system, based on sentinel units and severe acute respiratory infection notification.

\section{SP3-93 EFFECT OF THE DAY OF THE WEEK ON DIETARY CONSUMPTION ASSESSED BY FOOD FREOUENCY QUESTIONNAIRE}

doi:10.1136/jech.2011.1429760.93

${ }^{1} \mathrm{~S}$ L Rossato, ${ }^{1} \mathrm{R}$ L Henn, ${ }^{1,2}$ L B Moreira, ${ }^{1,2} \mathrm{~S}$ C Fuchs. ${ }^{1}$ Universidade Federal do Rio Grande do Sul, Porto Alegre, Brazil, ${ }^{2}$ Hospital de Clinicas de Porto Alegre, Porto Alegre, Brazil; ${ }^{3}$ Universidade do Vale do Rio dos Sinos, São Leopoldo, Brazil

Introduction The effect of the day of the week on nutrient intake on the overall consumption, collected by short reports, was notorious. However, it is still unknown for macronutrient intake collected by the food frequency questionnaire (FFQ).

Objective To evaluate the effect of data collect during the beginning and end of the week on macronutrient intake in data from FFO applied in adolescents from southern Brazil.

Methods A cross-sectional study investigated a population-based sample of 136 adolescents, aged 11-19 years. Dietary data were obtained by $\mathrm{FFO}$. Macronutrients intake was aggregated into 